\title{
Synthesis of 1-adamantyl alkyl ethers by intermolecular dehydration of 1-adamantanol with alcohols catalyzed by copper compounds
}

\author{
Ravil I. Khusnutdinov,* Alfiya R. Bayguzina, Rinat R. Mukminov, \\ Leisan I. Gimaletdinova, and Usein M. Dzhemilev \\ Institute of Petrochemistry and Catalysis of RAS, 141 Prospekt Oktyabrya, \\ 450075 Ufa, Bashkortostan, Russian Federation \\ E-mail:ink@anrb.ru
}

DOI: $\underline{\text { http://dx.doi.org/10.3998/ark.5550190.0015.400 }}$

\begin{abstract}
Adamantyl alkyl ethers were synthesized in high yields by intermolecular dehydration of 1-adamantanol with alcohols in the presence of copper-containing catalysts.
\end{abstract}

Keywords: 1-Adamantyl alkyl ethers, catalysis, intermolecular dehydration of alcohols, copper catalysts

\section{Introduction}

1-Adamantyl alkyl ethers are of considerable interest as additives that increase the oxidative stability and viscosity of lubricating oils, hydraulic and transmission fluids. ${ }^{1,2}$

Photolysis of mixtures of 1-haloadamantanes with alcohols in various solvents is the most widely used method for preparing 1-adamantyl alkyl ethers. The reaction is performed under irradiation using a low-pressure mercury lamp $(\lambda 254 \mathrm{~nm})$ for $24-48 \mathrm{~h}$ under nitrogen atmosphere. ${ }^{3-6}$ Another approach for the synthesis of 1-adamantyl methyl ether in 65-90\% yield involves the reactions of 1-chloro- or 1-iodoadamantanes with methanol in the presence of silver perchlorate. ${ }^{7}$ The reaction is partially reversible; therefore the precipitated $\mathrm{AgCl}$ is immediately filtered off. The excess of chloride ions is precipitated by adding silver perchlorate in acetone.

An interesting example of the synthesis of adamantyl benzyl ether is by reductive coupling of (1-adamantyloxy)trimethylsilane with benzaldehyde in the presence of trimethylsilane. ${ }^{8}$ The yield of adamantyl benzyl ether was $75 \%$. In another method, this ether can be prepared by benzylation of 1-adamantanol with 2-benzyloxy-1-methylpyridinium triflate (yield 80\%). ${ }^{9}$ 
Yet another method for the preparation of 1-adamantyl alkyl ethers is based on the reaction of 1-chloroadamantane with alcohols catalyzed by palladium complexes. ${ }^{10}$

This communication describes a convenient synthesis of 1-adamantyl alkyl ethers AdOR where $\mathrm{R}=\mathrm{CH}_{3}, \mathrm{C}_{2} \mathrm{H}_{5}, n-\mathrm{C}_{3} \mathrm{H}_{7}, n-\mathrm{C}_{4} \mathrm{H}_{9}, n-\mathrm{C}_{5} \mathrm{H}_{11}, n-\mathrm{C}_{8} \mathrm{H}_{17}$, cyclo- $\mathrm{C}_{5} \mathrm{H}_{9}$, and cyclo- $\mathrm{C}_{6} \mathrm{H}_{11}$, and 1-adamantyl benzyl ether by intermolecular dehydration of 1-adamantanol $\mathbf{1}$ with alcohols catalyzed by copper compounds.

\section{Results and Discussion}

The key regularities of the synthesis of 1-adamantyl alkyl ethers were studied in relation to the reaction of 1-adamantanol 1 with methanol in the presence of the following series of coppercontaining catalysts: $\mathrm{CuBr}_{2}, \mathrm{CuCl}_{2} \cdot 2 \mathrm{H}_{2} \mathrm{O}, \mathrm{Cu}(\mathrm{Acac})_{2}, \mathrm{Cu}\left(\mathrm{PhCO}_{2}\right)_{2} \cdot 2 \mathrm{H}_{2} \mathrm{O}$ (copper(II) benzoate), $\mathrm{Cu}\left(\mathrm{C}_{6} \mathrm{H}_{4}(\mathrm{OH}) \mathrm{CO}_{2}\right)_{2}$ (copper(II) salicylate), $\mathrm{Cu}(\mathrm{OAc})_{2}, \mathrm{CuI}$ and $\mathrm{Cu}(\mathrm{OAc})$, the best of which proved to be $\mathrm{CuBr}_{2}, \mathrm{CuCl}_{2} \cdot 2 \mathrm{H}_{2} \mathrm{O}$ and $\mathrm{Cu}\left(\mathrm{C}_{6} \mathrm{H}_{5} \mathrm{CO}_{2}\right)_{2} \cdot 2 \mathrm{H}_{2} \mathrm{O}$. The reaction was carried out at the following catalyst to reactant molar ratios: $[\mathrm{Cu}]:\left[1\right.$-adamantanol 1] : $\left[\mathrm{CH}_{3} \mathrm{OH}\right]=1-10: 100$ : 100-2000. The reaction temperatures were $200-240{ }^{\circ} \mathrm{C}$, the reaction times $1-6 \mathrm{~h}$ (Table 1 ).

Table 1. Synthesis of 1-adamantyl methyl ether $\mathbf{2}$ by the reaction of 1 -adamantanol $\mathbf{1}$ with methanol in the presence of copper-containing catalysts $\left(\mathrm{T}=220{ }^{\circ} \mathrm{C}\right.$ unless otherwise stated $)$

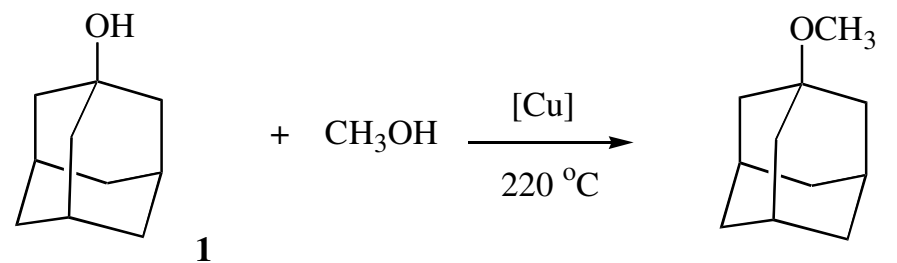

1

2

\begin{tabular}{|c|c|c|c|c|}
\hline Entry & {$[\mathrm{Cu}]:[\mathbf{1}]:\left[\mathrm{CH}_{3} \mathrm{OH}\right]$} & {$[\mathrm{Cu}]$} & $\begin{array}{l}\text { Time } \\
\text { (h) }\end{array}$ & $\begin{array}{c}\text { Yield } 2 \\
(\%)\end{array}$ \\
\hline 1 & $0: 100: 1000$ & - & 1 & 9 \\
\hline 2 & $1: 100: 1000$ & $\mathrm{CuBr}_{2}$ & 1 & 85 \\
\hline 3 & $1: 100: 1000$ & $\mathrm{Cu}(\mathrm{OAc})_{2}$ & 1 & 80 \\
\hline 4 & $1: 100: 1000$ & $\mathrm{CuCl}_{2} \cdot 2 \mathrm{H}_{2} \mathrm{O}$ & 1 & 85 \\
\hline 5 & $1: 100: 1000$ & $\mathrm{Cu}\left(\mathrm{PhCO}_{2}\right) \cdot 2 \mathrm{H}_{2} \mathrm{O}$ & 1 & 85 \\
\hline 6 & $1: 100: 1000$ & $\mathrm{CuI}$ & 1 & 55 \\
\hline 7 & $1: 100: 1000$ & $\mathrm{CuOAc}$ & 1 & 65 \\
\hline 8 & $1: 100: 1000$ & $\mathrm{Cu}\left[\mathrm{C}_{6} \mathrm{H}_{4}(\mathrm{OH}) \mathrm{CO}_{2}\right]_{2}$ & 1 & 70 \\
\hline 9 & $1: 100: 1000$ & $\mathrm{Cu}(\mathrm{Acac})_{2}$ & 1 & 80 \\
\hline 10 & $10: 100: 1000$ & $\mathrm{CuBr}_{2}$ & 1 & 70 \\
\hline
\end{tabular}


Table 1. Continued

\begin{tabular}{ccccc}
\hline Entry & {$[\mathrm{Cu}]:[1]:[\mathrm{CH} 3 \mathrm{OH}]$} & {$[\mathrm{Cu}]$} & $\begin{array}{c}\text { Time } \\
(\mathrm{h})\end{array}$ & $\begin{array}{c}\text { Yield 2 } \\
(\%)\end{array}$ \\
\hline 11 & $10: 100: 1000$ & $\mathrm{CuCl} 2 \cdot 2 \mathrm{H} 2 \mathrm{O}$ & 1 & 80 \\
12 & $10: 100: 1000$ & $\mathrm{Cu}(\mathrm{PhCO} 2) \cdot 2 \mathrm{H} 2 \mathrm{O}$ & 1 & 5 \\
13 & $10: 100: 1000$ & $\mathrm{Cu}(\mathrm{Acac})_{2}$ & 1 & 25 \\
14 & $1: 100: 100$ & $\mathrm{CuBr}_{2}$ & 1 & 50 \\
15 & $1: 100: 500$ & $\mathrm{CuBr}_{2}$ & 1 & 80 \\
16 & $1: 100: 750$ & $\mathrm{CuBr}_{2}$ & 1 & 80 \\
17 & $1: 100: 1500$ & $\mathrm{CuBr}_{2}$ & 1 & 85 \\
18 & $1: 100: 2000$ & $\mathrm{CuBr}_{2}$ & 1 & 85 \\
19 & $1: 100: 1000$ & $\mathrm{CuBr}_{2}$ & 2 & 85 \\
20 & $1: 100: 1000$ & $\mathrm{CuBr}_{2}$ & 4 & 80 \\
21 & $1: 100: 1000$ & $\mathrm{CuBr}_{2}$ & 6 & 79 \\
22 & $10: 100: 1000$ & $\mathrm{CuBr}_{2}$ & 4 & 36 \\
$23^{a}$ & $1: 100: 1000$ & $\mathrm{CuBr}_{2}$ & 1 & 75 \\
$24^{b}$ & $1: 100: 1000$ & $\mathrm{CuBr}_{2}$ & 1 & 80 \\
\hline${ }^{a} \mathrm{~T}=200{ }^{\circ} \mathrm{C} .{ }^{b} \mathrm{~T}=240{ }^{\circ} \mathrm{C}$ & & &
\end{tabular}

From the data presented in Table 1, it can be seen that the highest yield of 1-adamantyl methyl ether $\mathbf{2}$ of $85 \%$ is achieved when the intermolecular dehydration of 1-adamantanol 1 with methanol is carried out under the following conditions: $220{ }^{\circ} \mathrm{C}, 1 \mathrm{~h}$, and a $1: 100: 1000$ ratio of the catalyst, compound $\mathbf{1}$ and methanol (Table 1, entry 2).

Table 2. Synthesis of 1-adamantyl alkyl ethers ${ }^{a}$

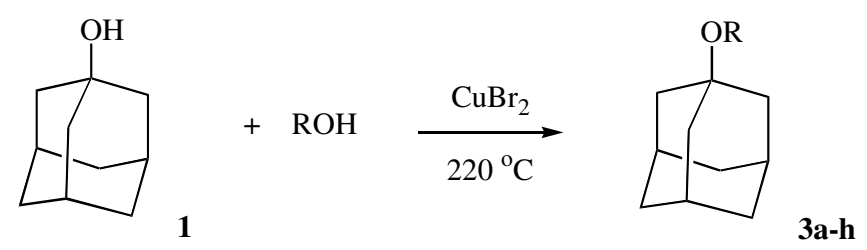

\begin{tabular}{cccc|cccc}
\hline Entry & ROH & AdOR & $\begin{array}{c}\text { Yield (\%) } \\
\text { 3(a-d) }\end{array}$ & Entry & ROH & AdOR & $\begin{array}{c}\text { Yield (\%) } \\
\text { 3(e-h) }\end{array}$ \\
\hline 1 & $\mathrm{C}_{2} \mathrm{H}_{5} \mathrm{OH}$ & 3a & 74 & 5 & $n-\mathrm{C}_{8} \mathrm{H}_{17} \mathrm{OH}$ & 3e & 24 \\
2 & $n-\mathrm{C}_{3} \mathrm{H}_{7} \mathrm{OH}$ & 3b & 65 & 6 & cyclo $-\mathrm{C}_{5} \mathrm{H}_{9} \mathrm{OH}$ & $\mathbf{3 f}$ & 41 \\
\hline
\end{tabular}


Table 2. Continued

\begin{tabular}{cccc|cccc}
\hline Entry & $R O H$ & AdOR & $\begin{array}{c}\text { Yield (\%) } \\
3(\mathrm{a}-\mathrm{d})\end{array}$ & Entry & ROH & AdOR & $\begin{array}{c}\text { Yield (\%) } \\
3(\mathrm{e}-\mathrm{h})\end{array}$ \\
\hline 3 & $n-\mathrm{C} 4 \mathrm{H} 9 \mathrm{OH}$ & $\mathbf{3 c}$ & 63 & 7 & cyclo-C6H11OH & $\mathbf{3 g}$ & 21 \\
& & & & & & & \\
4 & $n-\mathrm{C}_{5} \mathrm{H}_{11} \mathrm{OH}$ & $\mathbf{3 d}$ & 50 & 8 & $\mathrm{C}_{6} \mathrm{H}_{5} \mathrm{CH}_{2} \mathrm{OH}$ & $\mathbf{3 h}$ & 59 \\
\hline
\end{tabular}

${ }^{a}$ Reaction conditions: $220^{\circ} \mathrm{C}, 1 \mathrm{~h},\left[\mathrm{CuBr}_{2}\right]:[1]:[\mathrm{ROH}]=1: 100: 1000$

The reaction is general. Under the optimal conditions, it can be performed for a broad range of alcohols ROH, where $\mathrm{R}=\mathrm{C}_{2} \mathrm{H}_{5}, n-\mathrm{C}_{3} \mathrm{H}_{7}, n-\mathrm{C}_{4} \mathrm{H}_{9}, n-\mathrm{C}_{5} \mathrm{H}_{11}, n-\mathrm{C}_{8} \mathrm{H}_{17}$, cyclo- $\mathrm{C}_{5} \mathrm{H}_{9}$, cyclo- $\mathrm{C}_{6} \mathrm{H}_{11}$ and $\mathrm{C}_{6} \mathrm{H}_{5} \mathrm{CH}_{2}$ (Table 2).

It is worth noting that the use of a 10 -fold excess of high-boiling alcohols such as n-octanol, cyclopentanol, cyclohexanol or benzyl alcohol is undesirable, because it complicates the isolation of the desired adamantyl alkyl ethers by necessitating the removal of unreacted alcohols and homoethers (dioctyl, dicyclopentyl, dicyclohexyl or dibenzyl) from the reaction mixture.

In order to increase the conversion of alcohols $(\mathrm{ROH})$ and decrease the yield of side homoethers (ROR), the intermolecular dehydration of 1-adamantanol 1 with $n$-octanol, cyclopentanol, cyclohexanol and benzyl alcohol was carried out at an equimolar reactant ratio, which increased the yield of the target 1-adamantyl alkyl ethers and also increased the conversion of the alcohols (ROH) (Table 3).

Table 3. The reaction of 1-adamantanol with an equivalent amount of high-boiling alcohol

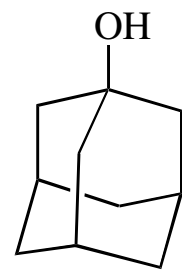

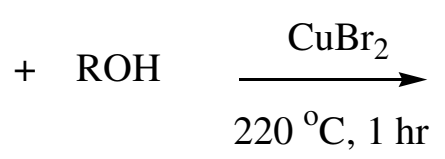

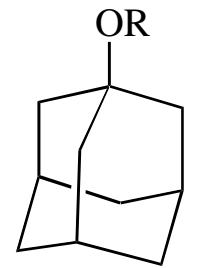

$$
+ \text { ROR }
$$

1

\section{3e-h}

$\left[\mathrm{CuBr}_{2}\right]:[1]:[\mathrm{ROH}]=1: 100: 100$

\begin{tabular}{ccccccc}
\hline Entry & \multicolumn{2}{c}{$\mathrm{ROH}$} & \multicolumn{2}{c}{ Conversion, \% } & \multicolumn{2}{c}{ Yield, \% } \\
& & $\mathbf{1}$ & $\mathrm{ROH}$ & $\mathbf{3 e - \mathbf { h } ^ { a }}$ & $\mathrm{ROR}^{b}$ \\
\hline 1 & $\mathrm{R}={ }_{n-\mathrm{C}_{8} \mathrm{H}_{17}}$ & 72 & 100 & 72 & $:$ & 1.5 \\
2 & & 32 & 100 & 32 & $:$ & 5 \\
\hline
\end{tabular}


Table 3. Continued

\begin{tabular}{|c|c|c|c|c|c|c|}
\hline \multirow[t]{2}{*}{ Entry } & \multirow[t]{2}{*}{$\mathrm{ROH}$} & \multicolumn{2}{|c|}{ Conversion, $\%$} & \multicolumn{3}{|c|}{ Yield, \% } \\
\hline & & 1 & $\mathrm{ROH}$ & $3 \mathbf{e}-\mathbf{h}^{a}$ & & $\mathrm{ROR}^{b}$ \\
\hline 3 & & 30 & 100 & 30 & : & 0 \\
\hline 4 & & 73 & 100 & 73 & : & 35 \\
\hline
\end{tabular}

${ }^{a}$ Yields are given relative to converted $\mathbf{1}$

${ }^{b}$ Yields are given relative to converted alcohol $(\mathrm{ROH})$

\section{Conclusions}

It was found that copper compounds $\mathrm{CuBr}_{2}, \mathrm{CuCl}_{2} \cdot 2 \mathrm{H}_{2} \mathrm{O}$ and $\mathrm{Cu}\left(\mathrm{PhCO}_{2}\right)_{2} \cdot 2 \mathrm{H}_{2} \mathrm{O}$ are effective catalysts of the synthesis of 1-adamantyl alkyl ethers by intermolecular dehydration of 1adamantanol and alcohols. In the presence of these catalysts, we performed the synthesis of the known 1-adamantyl methyl, ethyl, propyl and benzyl ethers and the previously unknown 1adamantyl butyl, octyl cyclopentyl, and cyclohexyl ethers.

\section{Experimental Section}

General. ${ }^{1} \mathrm{H}$ and ${ }^{13} \mathrm{C}$ NMR spectra were measured on a Bruker Avance-400 spectrometer (400.13 and $100.62 \mathrm{MHz}$, respectively) in $\mathrm{CDCl}_{3}$, the chemical shifts are referred to TMS. Mass spectra were run on a Shimadzu GCMS-QP2010Plus GC/MS spectrometer (an SPB-5 capillary column, $30 \mathrm{~m} \times 0.25 \mathrm{~mm}$, helium as a carrier gas, temperature programming from 40 to $300^{\circ} \mathrm{C}$ at $8{ }^{\circ} \mathrm{C} / \mathrm{min}$, evaporation temperature $280^{\circ} \mathrm{C}$, temperature of the ion source $200^{\circ} \mathrm{C}$, ionization energy $70 \mathrm{eV}$ ). Chromatographic analysis was carried out on a Shimadzu GC-9A, GC-2014 instrument [2 $\mathrm{m} \times 3 \mathrm{~mm}$ column, silicone SE-30 (5\%) on Chromaton N-AW-HMDS as the stationary phase, temperature programming from 50 to $270{ }^{\circ} \mathrm{C}$ at $8{ }^{\circ} \mathrm{C} / \mathrm{min}$, helium as the carrier gas $(47$ $\mathrm{mL} / \mathrm{min})]$.

The elemental composition of the samples was determined on a Carlo Erba 1106 elemental analyzer.

\section{General procedure for the synthesis of compounds $2,3 \mathrm{a}-\mathrm{h}$}

In an argon flow, the $10-\mathrm{mL}$ glass ampoule was charged with $\mathrm{CuBr}_{2}(0.013 \mathrm{mmol})$, 1adamantanol 1 (1.3 mmol), alcohol (13 mmol of methanol, ethanol, 1-propanol, 1-butanol, 1- 
pentanol or $1.3 \mathrm{mmol}$ of 1-octanol, cyclopentanol, cyclohexanol, benzyl alcohol). The sealed ampoule and appropriate alcohol $(2 \mathrm{~mL})$ to counterbalance the internal pressure of the reactant mixture when heated were placed in a $17-\mathrm{mL}$ stainless-steel micro autoclave, the autoclave was sealed and heated at $220{ }^{\circ} \mathrm{C}$ for $1 \mathrm{~h}$. After completion of the reaction, the autoclave was cooled to room temperature, the ampoule was opened, and the reaction mixture was filtered through a paper filter. The solvent was distilled off. The adamantyl alkyl ether was separated from the unreacted 1-adamantanol 1 by decantation in hexane. Alternatively, the mixture was separated by column chromatography on silica gel using a $1: 1$ hexane : benzene mixture for elution $(l=$ $21 \mathrm{~cm}, d=1.2 \mathrm{~cm})$.

Detailed NMR spectra are given in the Supplementary Data file.

1-Adamantyl methyl ether (2). ${ }^{10}$ Yield $85 \%$; colorless liquid; bp $67-68{ }^{\circ} \mathrm{C} / 3 \mathrm{~mm} .{ }^{1} \mathrm{H}$ NMR $\left(400.13 \mathrm{MHz}, \mathrm{CDCl}_{3}\right): \delta 3.11\left(\mathrm{~s}, 3 \mathrm{H}, \mathrm{OCH}_{3}\right), 2.05$ (br s, 3H, CH), $1.63\left(\mathrm{~s}, 6 \mathrm{H}, \mathrm{CH}_{2}\right), 1.51(\mathrm{~d}, J$ $\left.11.2 \mathrm{~Hz}, 6 \mathrm{H}, \mathrm{CH}_{2},\right) .{ }^{13} \mathrm{C} \mathrm{NMR}\left(100.62 \mathrm{MHz}, \mathrm{CDCl}_{3}\right): \delta 71.70,47.55,40.86,36.33,30.44$.

1-Adamantyl ethyl ether (3a). ${ }^{10}$ Yield 74\%; colorless liquid; bp 108-109 ${ }^{\circ} \mathrm{C} / 13 \mathrm{~mm} .{ }^{1} \mathrm{H} \mathrm{NMR}$ $\left(400.13 \mathrm{MHz}, \mathrm{CDCl}_{3}\right): \delta 3.46\left(\mathrm{q}, J 7.2 \mathrm{~Hz}, 2 \mathrm{H}, \mathrm{CH}_{2} \mathrm{CH}_{3}\right.$ ), 2.12 (br s, 3H, CH), 1.61 (d, J 6.8 Hz, $\left.6 \mathrm{H}, \mathrm{CH}_{2}\right), 1.61\left(\mathrm{~s}, 6 \mathrm{H}, \mathrm{CH}_{2}\right), 1.15\left(\mathrm{t}, J 6.8 \mathrm{~Hz}, 3 \mathrm{H}, \mathrm{CH}_{3}\right) .{ }^{13} \mathrm{C} \mathrm{NMR}\left(100.62 \mathrm{MHz}, \mathrm{CDCl}_{3}\right): \delta$ 71.78, 54.88, 41.62, 36.52, 30.50, 16.37.

1-Adamantyl $\boldsymbol{n}$-propyl ether (3b). ${ }^{10}$ Yield $65 \%$; colorless liquid; bp $90{ }^{\circ} \mathrm{C} / 2 \mathrm{~mm} .{ }^{1} \mathrm{H} \mathrm{NMR}$ (400.13 MHz, $\mathrm{CDCl}_{3}$ ): $\delta 3.62\left(\mathrm{t}, J 6.8 \mathrm{~Hz}, 2 \mathrm{H}, \mathrm{CH}_{2} \mathrm{CH}_{2}\right), 2.15$ (br s, $\left.3 \mathrm{H}, \mathrm{CH}\right), 1.76\left(\mathrm{~s}, 6 \mathrm{H}, \mathrm{CH}_{2}\right)$, $1.58\left(\mathrm{~d}, J 7.6 \mathrm{~Hz}, 6 \mathrm{H}, \mathrm{CH}_{2}\right), 0.09\left(\mathrm{t}, J 7.6 \mathrm{~Hz}, 3 \mathrm{H}, \mathrm{CH}_{3}\right) .{ }^{13} \mathrm{C} \mathrm{NMR}\left(100.62 \mathrm{MHz}, \mathrm{CDCl}_{3}\right): \delta$ 71.64, 61.47, 41.63, 36.40, 30.67, 23.91, 10.69 .

1-Adamantyl $\boldsymbol{n}$-butyl ether (3c). Yield 63\%; colorless liquid; bp 103-105 ${ }^{\circ} \mathrm{C} / 2 \mathrm{~mm} .{ }^{1} \mathrm{H}$ NMR (400.13 MHz, $\mathrm{CDCl}_{3}$ ): $\delta 3.38\left(\mathrm{t}, J 6.8 \mathrm{~Hz}, 2 \mathrm{H}, \mathrm{CH}_{2}\right.$ ), 2.12 (br s, $\left.3 \mathrm{H}, \mathrm{CH}\right), 1.73\left(\mathrm{~m}, 6 \mathrm{H}, \mathrm{CH}_{2}\right)$, $1.61\left(\mathrm{~m}, 6 \mathrm{H}, \mathrm{CH}_{2}\right.$ ), 1.49 (qui, 2H, $\mathrm{CH}_{2}$ ), 1.35 (sxt, $\left.2 \mathrm{H}, \mathrm{CH}_{2}\right), 0.9\left(\mathrm{t}, J 7.2 \mathrm{~Hz}, 3 \mathrm{H}, \mathrm{CH}_{3}\right) .{ }^{13} \mathrm{C}$ NMR (100.62 MHz, $\left.\mathrm{CDCl}_{3}\right): \delta 71.60,59.37,41.61,36.55,32.85,30.52,19.41,13.92$. Anal. Calcd for $\mathrm{C}_{14} \mathrm{H}_{24} \mathrm{O}$ : C, 80.71; H, 11.61. Found: 80.64; $\mathrm{H} 11.65 \%$.

1-Adamantyl $\boldsymbol{n}$-pentyl ether (3d). Yield 50\%; colorless liquid; bp 120-122 ${ }^{\circ} \mathrm{C} / 3 \mathrm{~mm} .{ }^{1} \mathrm{H}$ NMR (400.13 MHz, $\mathrm{CDCl}_{3}$ ): $\delta 3.37$ (t, J $6.8 \mathrm{~Hz}, 2 \mathrm{H}, \mathrm{CH}_{2}$ ), 2.12 (br s, $\left.3 \mathrm{H}, \mathrm{CH}\right), 1.73\left(\mathrm{~m}, 6 \mathrm{H}, \mathrm{CH}_{2}\right.$ ), $1.61\left(\mathrm{~m}, 6 \mathrm{H}, \mathrm{CH}_{2}\right), 1.50\left(\mathrm{~m}, 2 \mathrm{H}, \mathrm{CH}_{2}\right), 1.29-1.31\left(\mathrm{~m}, 4 \mathrm{H}, \mathrm{CH}_{2}\right), 0.88\left(\mathrm{t}, J 6.8 \mathrm{~Hz}, 3 \mathrm{H}, \mathrm{CH}_{3}\right) .{ }^{13} \mathrm{C}$ NMR (100.62 MHz, $\left.\mathrm{CDCl}_{3}\right): \delta$ 71.56, 59.73, 41.61, 36.55, 30.52, 30.46, 28.45, 22.57, 14.04. Anal. Calcd for $\mathrm{C}_{15} \mathrm{H}_{26} \mathrm{O}$ : C, 81.02; H, 11.79. Found: 81.05; H $11.59 \%$.

1-Adamantyl $\boldsymbol{n}$-octyl ether (3e). Yield 72\%; colorless liquid; bp $138-140{ }^{\circ} \mathrm{C} / 1 \mathrm{~mm} .{ }^{1} \mathrm{H}$ NMR (400.13 MHz, $\mathrm{CDCl}_{3}$ ): $\delta 3.39$ (t, J $6.8 \mathrm{~Hz}, 2 \mathrm{H}, \mathrm{CH}_{2}$ ), 2.14 (br s, 3H, CH), $1.75\left(\mathrm{~m}, 6 \mathrm{H}, \mathrm{CH}_{2}\right.$ ), $1.63\left(\mathrm{~m}, 6 \mathrm{H}, \mathrm{CH}_{2}\right), 1.52\left(\mathrm{~m}, 2 \mathrm{H}, \mathrm{CH}_{2}\right), 1.28-1.30\left(\mathrm{~m}, 10 \mathrm{H}, \mathrm{CH}_{2}\right), 0.89\left(\mathrm{t}, J 6.4 \mathrm{~Hz}, 3 \mathrm{H}, \mathrm{CH}_{3}\right) .{ }^{13} \mathrm{C}$ NMR (100.62 $\left.\mathrm{MHz} \mathrm{CDCl}_{3}\right): \delta 71.61,59.79,41.63,36.57,31.86,30.79,30.54,26.29,29.51$, 29.32, 22.67, 14.09. MS: m/z (\%) $264.30\left(\mathrm{M}^{+}, 1.79\right), 207.20$ (16.54), 136.20 (10.87), 135.20 (100.00), 110.15 (8.76), 95.10 (39.97), 79.10 (8.29). Anal. Calcd for $\mathrm{C}_{18} \mathrm{H}_{32} \mathrm{O}: \mathrm{C}, 81.75$; $\mathrm{H}$, 12.20. Found: $81.78 ; \mathrm{H} 12.14 \%$.

1-Adamantyl cyclopentyl ether (3f). Yield 41\%; colorless liquid; bp $112-114{ }^{\circ} \mathrm{C} / 1 \mathrm{~mm} .{ }^{1} \mathrm{H}$ NMR (400.13 MHz, $\mathrm{CDCl}_{3}$ ): $\delta 4.15(\mathrm{~m}, 1 \mathrm{H}, \mathrm{CH}), 2.14$ (br s, 3H, CH), $1.77\left(\mathrm{~m}, 2 \mathrm{H}, \mathrm{CH}_{2}\right), 1.75$ 
$\left(\mathrm{m}, 6 \mathrm{H}, \mathrm{CH}_{2}\right), 1.70\left(\mathrm{~m}, 2 \mathrm{H}, \mathrm{CH}_{2}\right), 1.62\left(\mathrm{~m}, 6 \mathrm{H}, \mathrm{CH}_{2}\right), 1.48\left(\mathrm{~m}, 2 \mathrm{H}, \mathrm{CH}_{2}\right), 1.46\left(\mathrm{~m}, 2 \mathrm{H}, \mathrm{CH}_{2}\right) .{ }^{13} \mathrm{C}$ NMR $\left(100.62 \mathrm{MHz}, \mathrm{CDCl}_{3}\right): \delta 72.08,71.53,42.69,36.54,35.07,30.60,23.67 . \mathrm{MS}: \mathrm{m} / \mathrm{z}(\%)$ $220.15\left(\mathrm{M}^{+}, 8.05\right), 136.20$ (10.79), 135.20 (100.00), 95.10 (9.74), 93.10 (10.71), 79.10 (10.58). Anal. Calcd for $\mathrm{C}_{15} \mathrm{H}_{24} \mathrm{O}: \mathrm{C}, 81.76 ; \mathrm{H}, 10.98$. Found: $81.82 ; \mathrm{H} 10.89 \%$.

1-Adamantyl cyclohexyl ether (3g). Yield 30\%; colorless liquid; bp 133-135 ${ }^{\circ} \mathrm{C} / 2 \mathrm{~mm} .{ }^{1} \mathrm{H}$ NMR (400.13 MHz, $\mathrm{CDCl}_{3}$ ): $\delta 3.49(\mathrm{~m}, 1 \mathrm{H}, \mathrm{CH}), 2.13$ (br s, 3H, CH), $1.75\left(\mathrm{~m}, 6 \mathrm{H}, \mathrm{CH}_{2}\right), 1.74$ $\left(\mathrm{m}, 4 \mathrm{H}, \mathrm{CH}_{2}\right), 1.62\left(\mathrm{~m}, 6 \mathrm{H}, \mathrm{CH}_{2}\right), 1.56\left(\mathrm{~m}, 2 \mathrm{H}, \mathrm{CH}_{2}\right), 1.27\left(\mathrm{~m}, 4 \mathrm{H}, \mathrm{CH}_{2}\right) .{ }^{13} \mathrm{C} \mathrm{NMR}(100.62$ $\left.\mathrm{MHz}, \mathrm{CDCl}_{3}\right): \delta 72.32,68.12,42.60,36.53,35.90,30.58,25.60,25.24 . \mathrm{MS}: \mathrm{m} / \mathrm{z}(\%) 234.20$ $\left(\mathrm{M}^{+}, 8.23\right), 136.20$ (11.06), 135.20 (100.00), 95.10 (16.05), 93.10 (8.19), 79.10 (8.60). Anal. Calcd for $\mathrm{C}_{16} \mathrm{H}_{26} \mathrm{O}$ : C, 81.99; H, 11.18. Found: 81.92; $\mathrm{H} 11.21 \%$.

1-Adamantyl benzyl ether (3h). ${ }^{8}$ Yield 73\%; colorless liquid; bp 137-140 ${ }^{\circ} \mathrm{C} / 1 \mathrm{~mm} .{ }^{1} \mathrm{H}$ NMR (400.13 MHz, $\mathrm{CDCl}_{3}$ ): $\delta$ 7.21-7.42 (m, 5H, $\mathrm{C}_{6} \mathrm{H}_{5}$ ), 4.56 (s, 2H, $\mathrm{CH}_{2}$ ), 2.23 (br s, 3H, CH), 1.91 $\left(\mathrm{m}, 6 \mathrm{H}, \mathrm{CH}_{2}\right), 1.71\left(\mathrm{~m}, 6 \mathrm{H}, \mathrm{CH}_{2}\right) .{ }^{13} \mathrm{C} \mathrm{NMR}\left(100.62 \mathrm{MHz}, \mathrm{CDCl}_{3}\right): \delta 140.22,128.28,127.49$, 127.07, 72.77, 62.37, 41.81, 36.57, 30.66. MS: m/z (\%) 242.20 ( $\left.\mathrm{M}^{+}, 28.67\right), 185.10$ (12.20), 135.20 (58.04), 107.15 (7.18), 93.10 (13.31), 92.10 (10.03), 91.10 (100.00). Anal. Calcd for $\mathrm{C}_{17} \mathrm{H}_{22} \mathrm{O}: \mathrm{C}, 84.25 ; \mathrm{H}, 9.15$. Found: 84.17; H $9.18 \%$.

\section{Acknowledgements}

This work was performed under financial support from the Ministry of Education and Science of the Russian Federation (State contract no. 02.740.11.0631).

\section{References}

1. Bagrii, E. I. Adamantanes: Production, properties, application [in Russian]; Ed.; Nauka: Moscow, 1989; p 264.

2. Fort, R. C. Adamantane: The Chemistry of Diamond Molecules; Ed.; Marcel Dekker: New York, 1976; p 357.

3. Kropp, P. J.; Poindexter, G. S.; Pienta, N. J.; Hamilton, D. C. J. Am. Chem. Soc. 1976, 98, 8135 . http://dx.doi.org/10.1021/ja00441a043

4. Perkins, R. R.; Fincock, R. E. Tetrahedron Lett. 1975, 16, 943. http://dx.doi.org/10.1016/S0040-4039(00)72026-0

5. Miller, J. B.; Salvador, J. R. J. Org. Chem. 2002, 67, 435. http://dx.doi.org/10.1021/jo015896k

6. Kropp, P. J.; Adkins, R. L. J. Am. Chem. Soc. 1991, 113, 2709. http://dx.doi.org/10.1021/ja00007a053

7. Kevill, D. N.; Kolwysk, K. C.; Weite, F. L. J. Am. Chem. Soc. 1970, 92, 7300. 
http://dx.doi.org/10.1021/ja00728a012

8. Hartz, N.; Prakash, G. K. S.; Olah, G. A. Synlett 1992, 7, 569.

http://dx.doi.org/10.1055/s-1992-21418

9. Poon, K. W. C.; Dudley, G. B. J. Org. Chem. 2006, 71, 3923.

http://dx.doi.org/10.1021/jo0602773

10. Khusnutdinov, R. I.; Shchadneva, N. A.; Malikov, A. I.; Dzhemilev, U. M. Petroleum Chemistry 2000, 40, 419. 